\title{
21. CARBON AND CARBONATE ANALYSES, LEG 38
}

\author{
Kenneth Thompson, Scripps Institution of Oceanography, La Jolla, California
}

Leg $38 \mathrm{CaCO}_{3}$ samples were collected onboard ship in 3-cc vials at the time the core was split open. On shore the samples were dried and ground to a homogeneous powder. The ground sediment was redried at $105^{\circ}-110^{\circ} \mathrm{C}$ and two samples from each vial. a 0.1 -g and 0.5 -g sample, were then weighed into LECO crucibles. The 0.5 -g sample was acidified with $10 \% \mathrm{HCl}$, washed with distilled $\mathrm{H}_{2} \mathrm{O}$, redried and analyzed for acid insoluble (organic) carbon using a LECO WR-12 analyzer. The $0.1 \mathrm{~g}$ sample was treated only with distilled water to cake the sample before analysis for total carbon. If the results showed less than $10 \% \mathrm{CaCO}_{3}$, an additional 0.5 -g sample was analyzed for greater accuracy. The $\mathrm{CaCO}_{3}$ percentages were calculated as follows: $(\%$ total $\mathrm{C}-\%$ organic $\mathrm{C}) \times 8.33=\% \mathrm{CaCO}_{3}$. Although other carbonates may be present, all acidsoluble carbon was calculated as $\mathrm{CaCO}_{3}$. All weights are given in weight percent.

Detailed descriptions of technique and theory are in Volume 4 of the Initial Reports of DSDP (Bader, Gerard, et al., 1970).

For control purposes a sediment standard was made up from Deep Sea Drilling material and analyzed for total carbon at predetermined intervals with the regular samples. The carbonate content of these standards was determined by commercial laboratories. ${ }^{1}$ Listed below are the statistical data for these standards.

\begin{tabular}{ccccc}
\hline $\begin{array}{c}\text { DSDP } \\
\text { Std. }\end{array}$ & $\begin{array}{c}\text { No. of } \\
\text { Samples }\end{array}$ & $\begin{array}{c}\text { Totals as } \\
\mathrm{CaCO}_{3}(\%)\end{array}$ & $\begin{array}{c}\text { Standard } \\
\text { Deviation } \\
(\%)\end{array}$ & $\begin{array}{c}\text { Maximum } \\
\text { Range } \\
(\%)\end{array}$ \\
\hline 7 & 13 & 51.13 & 0.39 & 1.33 \\
9 & 23 & 26.79 & 0.28 & 1.13 \\
\hline
\end{tabular}

These data indicate the precision of the mechanical aspects of LECO analysis and do not necessarily reflect the precision of the total analytical procedure. This may be affected by factors such as sample homogeneity or contamination during sample preparation.

The carbon determinations were made using a LECO carbon analyzer which is described in detail in the LECO manual.

The LECO WR-12 selectively absorbs $\mathrm{CO}_{2}$ on a molecular sieve. Later, through heating, the $\mathrm{CO}_{2}$ is released and measured by its thermal conductivity. A sample of known amount is placed in a ceramic vial to which is added one or more combustibles such as ... (copper and iron chips). The crucible containing the sample is placed into a LECO high-frequency induction furnace within a combustion tube through which $\mathrm{O}_{2}$ is passed.

\footnotetext{
'Schwarzkopf Microanalytical Laboratory.
}

TABLE 1

Carbon and Carbonate Analyses, Leg 38

\begin{tabular}{|c|c|c|c|c|}
\hline $\begin{array}{c}\text { Sample } \\
\text { (Interval in } \mathrm{cm} \text { ) }\end{array}$ & Depth (m) & $\begin{array}{l}\text { Total } \\
\text { Carbon }\end{array}$ & $\begin{array}{l}\text { Organic } \\
\text { Carbon }\end{array}$ & $\mathrm{CaCO}_{3}$ \\
\hline \multicolumn{5}{|l|}{ Site 336} \\
\hline $2-4,70$ & 12.2 & 1.5 & 0.1 & 11 \\
\hline $3-2,110$ & 19.1 & 1.4 & 0.9 & 4 \\
\hline $5-1,40$ & 35.9 & 1.3 & 0.6 & 5 \\
\hline $5-6,40$ & 43.4 & 0.9 & 0.6 & 3 \\
\hline $8-1,30$ & 64.4 & 0.5 & 0.4 & 1 \\
\hline $8-5,86$ & 71.0 & 0.3 & 0.3 & 0 \\
\hline $9-2,78$ & 75.8 & 0.2 & 0.2 & 0 \\
\hline $9-5,75$ & 80.3 & 2.3 & 0.5 & 15 \\
\hline $10-2,69$ & 94.7 & 0.2 & 0.2 & 0 \\
\hline $11-3,80$ & 115.3 & 0.2 & 0.2 & 0 \\
\hline $12-2,83$ & 132.8 & 0.3 & 0.3 & 0 \\
\hline $15-1,80$ & 169.3 & 0.9 & 0.2 & 6 \\
\hline $16-5,60$ & 184.6 & 0.8 & 0.7 & 1 \\
\hline $18-2,30$ & 198.8 & 0.3 & 0.3 & 0 \\
\hline $18-3,20$ & 200.2 & 0.4 & 0.4 & 0 \\
\hline $22-6,0$ & 243.0 & 0.4 & 0.4 & 0 \\
\hline $23-3,48$ & 248.0 & 0.5 & 0.4 & 0 \\
\hline $24-3,90$ & 258.3 & 0.5 & 0.4 & 1 \\
\hline $25-3,73$ & 267.6 & 0.4 & 0.4 & 0 \\
\hline $26-3,78$ & 277.3 & 0.3 & 0.3 & 0 \\
\hline $27-3,81$ & 286.8 & 0.4 & 0.0 & 3 \\
\hline $29-2,70$ & 313.2 & 0.4 & 0.0 & 4 \\
\hline $30-1,30$ & 330.7 & 0.5 & 0.5 & 1 \\
\hline $30-3,46$ & 333.9 & 0.5 & 0.5 & 0 \\
\hline $30-3,54$ & 334.0 & 3.6 & 0.4 & 27 \\
\hline $30-3,60$ & 334.0 & 7.3 & 0.2 & 59 \\
\hline $35-3,63$ & 428.6 & 0.8 & 0.8 & 0 \\
\hline $36-3 ; 18$ & 437.7 & 0.9 & 0.7 & 1 \\
\hline $36-6,123$ & 443.2 & 0.5 & 0.5 & 0 \\
\hline $38-1,138$ & 468.9 & 0.1 & 0.1 & 0 \\
\hline $39-1,83$ & 473.3 & 0.1 & 0.0 & 0 \\
\hline $39-3,79$ & 476.3 & 0.1 & 0.0 & 0 \\
\hline $39-5,67$ & 479.2 & 0.1 & 0.1 & 0 \\
\hline \multicolumn{5}{|l|}{ Site 337} \\
\hline $1-2,44$ & 1.9 & 3.4 & 0.2 & 27 \\
\hline $1-5,47$ & 6.5 & 1.1 & 0.3 & 6 \\
\hline $2-3,22$ & 12.5 & 0.9 & 0.1 & 6 \\
\hline $3-2,110$ & 21.6 & 0.4 & 0.2 & 1 \\
\hline $4-2,50$ & 30.0 & 0.2 & 0.2 & 0 \\
\hline $5-2,122$ & 40.2 & 6.5 & 0.1 & 53 \\
\hline $5-4,125$ & 43.3 & 0.1 & 0.0 & 1 \\
\hline $6-2,83$ & 49.3 & 0.1 & 0.1 & 0 \\
\hline $6-2,101$ & 49.5 & 0.1 & 0.0 & 0 \\
\hline $10-3,39$ & 88.6 & 0.1 & 0.0 & 1 \\
\hline $11-3,99$ & 98.5 & 0.1 & 0.0 & 1 \\
\hline $12-3,30$ & 107.3 & 0.1 & 0.0 & 1 \\
\hline \multicolumn{5}{|l|}{ Site 338} \\
\hline $1-1,132$ & 1.3 & 0.9 & 0.5 & 3 \\
\hline $1-3,78$ & 3.8 & 1.8 & 0.2 & 13 \\
\hline $2-3,119$ & 13.7 & 1.9 & 0.3 & 13 \\
\hline $3-2,105$ & 21.6 & 1.1 & 0.8 & 2 \\
\hline 44,129 & 34.3 & 1.9 & 0.2 & 14 \\
\hline $5-5,86$ & 44.9 & 0.3 & 0.2 & 1 \\
\hline $6-5,50$ & 54.0 & 0.3 & 0.2 & 1 \\
\hline $7-1,105$ & 67.6 & 1.4 & 1.3 & 1 \\
\hline
\end{tabular}


TABLE 1 - Continued

\begin{tabular}{|c|c|c|c|c|}
\hline $\begin{array}{c}\text { Sample } \\
\text { (Interval in } \mathrm{cm} \text { ) }\end{array}$ & Depth (m) & $\begin{array}{l}\text { Total } \\
\text { Carbon }\end{array}$ & $\begin{array}{l}\text { Organic } \\
\text { Carbon }\end{array}$ & $\mathrm{CaCO}_{3}$ \\
\hline \multicolumn{5}{|c|}{ Site 338 - Continued } \\
\hline $13-5,50$ & 130.0 & 1.1 & 0.9 & 2 \\
\hline $15-3,75$ & 146.3 & 1.4 & 1.2 & 2 \\
\hline $17-4,39$ & 166.4 & 1.4 & 1.1 & 2 \\
\hline $18-1,79$ & 171.8 & 0.8 & 0.7 & 1 \\
\hline $19-1,137$ & 181.9 & 1.0 & 0.8 & 2 \\
\hline $19-5,73$ & 187.2 & 0.6 & 0.6 & 0 \\
\hline $20-1,142$ & 191.4 & 1.2 & 0.4 & 7 \\
\hline $20-5,75$ & 196.8 & 0.9 & 0.5 & 3 \\
\hline $21-1,125$ & 200.8 & 0.3 & 0.4 & 0 \\
\hline $22-1,74$ & 209.7 & 5.7 & 0.4 & 44 \\
\hline $22-4,85$ & 214.4 & 2.0 & 0.5 & 13 \\
\hline $22-6,138$ & 217.9 & 0.4 & 0.4 & 0 \\
\hline $23-1,73$ & 219.5 & 4.3 & 0.2 & 34 \\
\hline $23-6,107$ & 227.4 & 4.7 & 0.2 & 38 \\
\hline $24-3,60$ & 231.6 & 6.1 & 0.1 & 50 \\
\hline $26-3,140$ & 251.4 & 0.1 & 0.1 & 0 \\
\hline $27-5,89$ & 263.4 & 0.6 & 0.5 & 1 \\
\hline $30-4,61$ & 290.1 & 0.2 & 0.2 & 0 \\
\hline $31-1,80$ & 295.3 & 0.4 & 0.4 & 0 \\
\hline $32-4,27$ & 308.8 & 0.7 & 0.6 & 2 \\
\hline $33-4,50$ & 318.5 & 0.5 & 0.4 & 1 \\
\hline $35-2,45$ & 334.5 & 0.6 & 0.4 & 1 \\
\hline $37-2,64$ & 353.6 & 0.7 & 0.5 & 2 \\
\hline \multicolumn{5}{|l|}{ Site 339} \\
\hline $1-5,100$ & 7.0 & 2.1 & 0.5 & 14 \\
\hline $2-2,49$ & 10.0 & 2.2 & 0.5 & 14 \\
\hline $2-4,89$ & 13.4 & 1.2 & 0.4 & 7 \\
\hline $4-3,69$ & 30.7 & 2.1 & 0.6 & 12 \\
\hline $5-2,76$ & 38.8 & 1.4 & 0.6 & 6 \\
\hline $6-2,90$ & 48.4 & 2.0 & 0.7 & 10 \\
\hline $7-2,100$ & 58.0 & 0.6 & 0.4 & 2 \\
\hline $8-2,60$ & 67.1 & 1.5 & 0.6 & 8 \\
\hline $8-5,70$ & 71.7 & 0.4 & 0.4 & 1 \\
\hline $10-3,95$ & 88.0 & 0.3 & 0.0 & 3 \\
\hline $12-3,60$ & 106.6 & 0.6 & 0.6 & 0 \\
\hline \multicolumn{5}{|l|}{ Site 340} \\
\hline $1-3,93$ & 3.9 & 2.9 & 0.4 & 21 \\
\hline $6-2,27$ & 49.3 & 0.3 & 0.3 & 0 \\
\hline $8-4,50$ & 71.5 & 0.2 & 0.2 & 0 \\
\hline $9-3,15$ & 79.2 & 0.3 & 0.3 & 0 \\
\hline
\end{tabular}

Site 341

\begin{tabular}{lrrrr}
$1-2,8$ & 1.9 & 0.9 & 0.3 & 5 \\
$6-2,70$ & 49.7 & 1.3 & 0.6 & 6 \\
$7-2,30$ & 58.8 & 0.6 & 0.5 & 1 \\
$8-4,40$ & 71.4 & 1.4 & 0.6 & 7 \\
$10-4,69$ & 90.7 & 1.5 & 0.4 & 9 \\
$11-1,75$ & 95.8 & 1.4 & 0.4 & 8 \\
$12-4,10$ & 109.1 & 1.5 & 0.4 & 9 \\
$16-1,99$ & 162.5 & 1.2 & 0.3 & 8 \\
$20-1,120$ & 238.9 & 1.3 & 0.3 & 8 \\
$21-2,30$ & 258.8 & 2.1 & 0.4 & 15 \\
$23-6,55$ & 312.3 & 0.4 & 0.4 & 1 \\
$16-3,8$ & 355.0 & 4.7 & 0.0 & 39 \\
$27-3,82$ & 374.8 & 2.0 & 0.0 & 17 \\
$28-3,79$ & 393.7 & 1.5 & 0.0 & 13 \\
$29-1,120$ & 400.5 & 1.7 & 0.0 & 14 \\
$29-5,52$ & 405.8 & 3.3 & 0.0 & 28 \\
$32-2,113$ & 430.4 & 1.6 & 0.0 & 13 \\
$33-3,99$ & 441.4 & 1.7 & 1.5 & 2 \\
$34-3,80$ & 450.6 & 1.8 & 1.8 & 0 \\
Site 342 & & & & \\
$1-4,80$ & & & & \\
\hline
\end{tabular}

TABLE 1 - Continued

\begin{tabular}{lrrrr}
\hline $\begin{array}{c}\text { Sample } \\
\text { (Interval in cm) }\end{array}$ & Depth (m) & $\begin{array}{c}\text { Total } \\
\text { Carbon }\end{array}$ & $\begin{array}{l}\text { Organic } \\
\text { Carbon }\end{array}$ & $\mathrm{CaCO}_{3}$ \\
\hline Site 342-Continued & & & & \\
$2-2,90$ & 39.9 & 0.3 & 0.2 & 0 \\
$3-2,110$ & 87.6 & 0.3 & 0.3 & 0 \\
$4-2,84$ & 125.3 & 0.7 & 0.0 & 6 \\
$6-2,129$ & 144.8 & 1.1 & 0.3 & 7
\end{tabular}

Site 343

3-2, 75

4-2, 75

$4-4,70$

5-2, 78

6-1, 110

$7-3,60$

$8-3,30$

$10-2,40$

$11-2,70$

$15-2,110$

$16-1,123$
52.8

100.3

103.2

147.8

194.1

206.5

215.3

232.9

242.7

271.6

279.7

$\begin{array}{llr}1.7 & 0.5 & 10 \\ 0.5 & 0.3 & 2 \\ 2.4 & 0.1 & 19 \\ 0.8 & 0.0 & 6 \\ 1.1 & 0.0 & 10 \\ 0.4 & 0.3 & 1 \\ 1.2 & 0.4 & 7 \\ 0.9 & 0.5 & 3 \\ 0.4 & 0.3 & 0 \\ 1.0 & 0.8 & 2 \\ 1.2 & 0.0 & 10\end{array}$

Site 344

$2-2,20$

3-3, 50

5-3, 20

6-3, 110

$7-4,69$

$8-4,55$

$9-3,74$

$10-3,9$

$12-3,20$

$13-1,60$

$14-1,60$

$15-3,60$

$16-2,60$

$17-1,70$

$18-1,70$

21-2, 70

23-2, 70

24-1, 69

25-1, 65

26-1, 85

27-2, 50

28-2, 60

$30-1,120$

$32-2,74$

$33-2,48$

3.2
14.5
33.5
43.6
54.2
63.6
72.2
80.6
99.7
106.6
116.1
128.9
136.6
144.7
154.2
184.2
203.2
211.2
220.7
230.4
241.0
260.1
287.7
345.7
374.0

$\begin{array}{rrr}1.2 & 0.9 & 2 \\ 1.3 & 1.1 & 2 \\ 1.0 & 0.2 & 6 \\ 0.8 & 0.6 & 1 \\ 0.9 & 0.4 & 4 \\ 1.1 & 1.0 & 1 \\ 0.6 & 0.6 & 0 \\ 0.6 & 0.6 & 0 \\ 0.5 & 0.5 & 0 \\ 0.6 & 0.6 & 0 \\ 1.0 & 0.9 & 1 \\ 1.2 & 1.0 & 2 \\ 0.9 & 0.7 & 2 \\ 1.1 & 0.8 & 2 \\ 0.9 & 0.8 & 1 \\ 1.5 & 1.2 & 2 \\ 0.9 & 0.0 & 8 \\ 0.9 & 0.8 & 1 \\ 1.1 & 0.9 & 1 \\ 1.2 & 1.1 & 1 \\ 1.4 & 1.1 & 2 \\ 1.1 & 0.9 & 2 \\ 1.7 & 0.5 & 10 \\ 1.0 & 0.9 & 1 \\ 1.7 & 1.5 & 1\end{array}$

Site 345

1-2, 60

2-3, 70

3-2, 30

4-2, 69

5-2, 130

6-2, 50

$7-2,82$

8- 3,81

$9-3,85$

10-3, 40

$11-3,40$

14-3, 10

$16-4,35$

$17-3,68$

19-2, 75

20-3, 20

21-3, 8

23-4, 35

24-3, 35

25-2, 123

\begin{tabular}{rrrr}
2.1 & 2.4 & 0.3 & 17 \\
11.7 & 1.6 & 1.3 & 3 \\
19.3 & 4.6 & 0.2 & 36 \\
29.2 & 0.4 & 0.2 & 2 \\
39.3 & 0.7 & 0.7 & 0 \\
57.5 & 0.2 & 0.2 & 0 \\
76.8 & 0.3 & 0.3 & 0 \\
97.3 & 0.4 & 0.4 & 0 \\
116.4 & 0.4 & 0.4 & 0 \\
135.4 & 0.4 & 0.4 & 0 \\
163.8 & 0.4 & 0.4 & 0 \\
248.6 & 0.5 & 0.5 & 0 \\
335.9 & 0.6 & 0.0 & 5 \\
372.7 & 0.7 & 0.4 & 3 \\
447.8 & 0.4 & 0.3 & 1 \\
486.2 & 0.4 & 0.3 & 0 \\
524.1 & 0.4 & 0.3 & 0 \\
602.4 & 0.4 & 0.4 & 1 \\
638.8 & 0.4 & 0.3 & 1 \\
675.9 & 0.7 & 0.4 & 2 \\
\hline
\end{tabular}


TABLE 1 - Continued

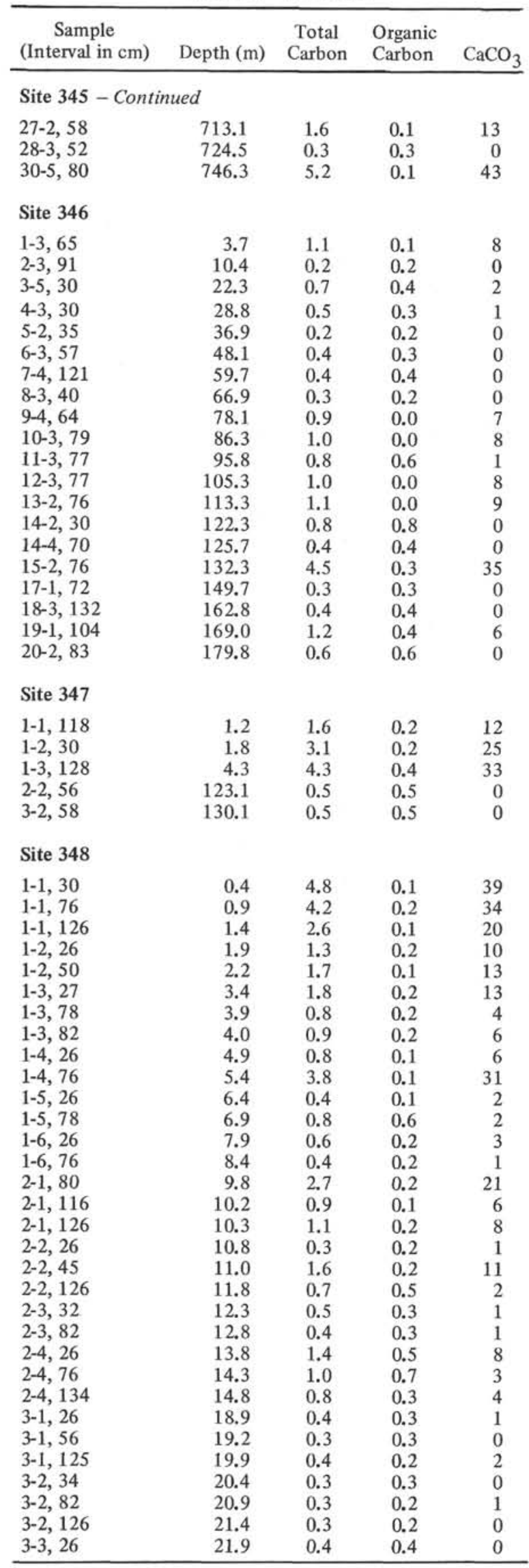

TABLE 1 - Continued

\begin{tabular}{ccccc}
\hline $\begin{array}{c}\text { Sample } \\
\text { (Interval in cm) }\end{array}$ & Depth $(\mathrm{m})$ & $\begin{array}{c}\text { Total } \\
\text { Carbon }\end{array}$ & $\begin{array}{c}\text { Organic } \\
\text { Carbon }\end{array}$ & $\mathrm{CaCO}_{3}$ \\
\hline
\end{tabular}

\section{Site 348 - Continued}

3-3, 73

$3-3,126$

$3-4,26$

3-4, 82

$3-4,132$

$3-5,26$

$3-5,76$

3-5, 118

$3-6,26$

3-6, 82

$4-1,76$

4-2, 26

4-2, 76

$4-2,123$

4-3, 71

4-3, 126

$5-2,26$

$5-2,76$

$6-2,0$

6-2, 0

6-3, 0

6-4, 0

$6-5,0$

$7-3,0$

$8-3,0$

9-3, 0

11-3, 0

$12-3,10$

$14-4,10$

$15-2,45$

$16-3,25$

$19-5,15$

20-2, 45

21-2, 90

23-3, 70

24-3, 56

26-3, 14

$27-2,42$

29-4, 16

$30-3,39$

$31-3,68$

22.3

22.9

23.4
23.9

23.9
24.4

24.4
24.9

25.4

25.8

26.4
26.9

38.3

39.3

39.8

40.2

41.2

41.8

58.3

58.8

67.5

67.5

69.0

70.5

72.0
78.5

97.5

116.5

154.5

164.1

194.5

210.5

231.2

271.7

277.3

287.4

326.2

345.4

376.1

401.6

429.4

489.2

506.4

516.6

$0.3 \quad 0.3$

$\begin{array}{ll}0.4 & 0.3\end{array}$

$\begin{array}{ll}0.4 & 0.3\end{array}$

$\begin{array}{ll}0.5 & 0.4\end{array}$

$\begin{array}{ll}0.3 & 0.3 \\ 0.3 & 0.2\end{array}$

$\begin{array}{ll}0.4 & 0.3\end{array}$

$0.3 \quad 0.2$

$\begin{array}{ll}0.2 & 0.2\end{array}$

$\begin{array}{ll}0.4 & 0.4 \\ 0.4 & 0.4\end{array}$

$\begin{array}{ll}0.4 & 0.3\end{array}$

$\begin{array}{ll}0.3 & 0.3\end{array}$

$\begin{array}{ll}0.2 & 0.2 \\ 0.3 & 0.3\end{array}$

$\begin{array}{ll}0.2 & 0.2\end{array}$

$\begin{array}{ll}0.3 & 0.3\end{array}$

$\begin{array}{ll}0.4 & 0.3\end{array}$

$\begin{array}{ll}0.3 & 0.2\end{array}$

$\begin{array}{ll}0.3 & 0.3 \\ 0.3 & 0.3\end{array}$

$\begin{array}{ll}0.3 & 0.2\end{array}$

1.9

0.3

0.3

$\begin{array}{ll}0.3 & 0.2\end{array}$

0.4

0.5

0.4

0.6
0.6

0.2

0.3

0.3

0.4

0.7
0.7

0.7

0.7
0.7

0.7

0.9
0.7

0.4

0.0
0.4

0.4
0.4

0.6

0.5

0.2

0.2

0.3

0.6
0.6

0.6

0.6

0.8

Site 349

1-3, 40

2-3, 44

3-2, 94

4-1, 85

5-3, 65

6-3, 60

$7-3,100$

9-2, 37

$10-4,61$

10-5, 7

11-2, 56

$12-5,128$

13-3, 123

3.4
57.2

93.9
101.9

101.9
114.5

123.6

133.5

150.4

191.6

192.6

226.6

269.8

314.2

1.6
0.3
0.4
0.3
0.2
1.3
1.0
1.0
1.0
1.1
0.7
0.9
0.4

$\begin{array}{rr}0.3 & 11 \\ 0.2 & 0 \\ 0.4 & 0 \\ 0.3 & 0 \\ 0.2 & 0 \\ 1.0 & 2 \\ 0.4 & 5 \\ 1.0 & 0 \\ 0.7 & 3 \\ 0.9 & 1 \\ 0.6 & 1 \\ 0.7 & 2 \\ 0.3 & 0\end{array}$

Site 350

$\begin{array}{rrrrr}1-2,65 & 2.2 & 3.5 & 0.1 & 28 \\ 2-3,56 & 30.6 & 0.4 & 0.3 & 1\end{array}$

Hole 352

\begin{tabular}{lrrrr}
$1-1,126$ & 1.3 & 2.1 & 0.1 & 16 \\
$1-2,126$ & 2.8 & 1.3 & 0.1 & 10 \\
$1-3,84$ & 3.8 & 1.1 & 0.1 & 8 \\
\hline
\end{tabular}


TABLE 1 - Continued

\begin{tabular}{|c|c|c|c|c|}
\hline $\begin{array}{c}\text { Sample } \\
\text { (Interval in } \mathrm{cm} \text { ) }\end{array}$ & Depth (m) & $\begin{array}{l}\text { Total } \\
\text { Carbon }\end{array}$ & $\begin{array}{l}\text { Organic } \\
\text { Carbon }\end{array}$ & $\mathrm{CaCO}_{3}$ \\
\hline \multicolumn{5}{|c|}{ Hole 352 - Continued } \\
\hline $\begin{array}{l}1-4,84 \\
1-5,84 \\
1-6,82 \\
2-1,84 \\
2-3,130 \\
2-4,60 \\
2-5,40 \\
4-2,93 \\
4-3,87 \\
4-4,84 \\
4-5,87 \\
4-6,84 \\
5-2,63\end{array}$ & $\begin{array}{r}5.3 \\
6.8 \\
8.3 \\
9.3 \\
12.8 \\
13.6 \\
14.9 \\
30.3 \\
31.7 \\
33.2 \\
34.7 \\
36.2 \\
39.1\end{array}$ & $\begin{array}{l}1.5 \\
1.7 \\
1.0 \\
0.7 \\
1.2 \\
0.9 \\
0.6 \\
0.8 \\
0.3 \\
0.4 \\
0.9 \\
0.4 \\
0.4\end{array}$ & $\begin{array}{l}0.1 \\
0.2 \\
0.2 \\
0.2 \\
0.2 \\
0.2 \\
0.2 \\
0.3 \\
0.2 \\
0.1 \\
0.2 \\
0.2 \\
0.1\end{array}$ & $\begin{array}{r}11 \\
12 \\
7 \\
4 \\
9 \\
6 \\
3 \\
4 \\
1 \\
2 \\
6 \\
2 \\
2\end{array}$ \\
\hline \multicolumn{5}{|l|}{ Hole 352A } \\
\hline $\begin{array}{l}1-1,142 \\
1-2,84 \\
2-1,129 \\
3-1,63 \\
3-2,71\end{array}$ & $\begin{array}{r}95.4 \\
96.3 \\
104.8 \\
113.6 \\
115.2\end{array}$ & $\begin{array}{l}6.3 \\
5.0 \\
5.3 \\
0.7 \\
7.1\end{array}$ & $\begin{array}{l}0.1 \\
0.1 \\
0.1 \\
0.1 \\
0.1\end{array}$ & $\begin{array}{r}52 \\
41 \\
43 \\
5 \\
58\end{array}$ \\
\hline
\end{tabular}

The carbon in the sample is converted to $\mathrm{CO}_{2}$ at temperatures in excess of $1000^{\circ} \mathrm{C}$. Metal oxides either remain in the crucible or are filtered out in a series of dust traps while sulfur gases are absorbed in a trap containing manganese dioxide. A heated catalyst (containing rare earth and copper oxides) converts any $\mathrm{CO}$ to $\mathrm{CO}_{2}$. Moisture is removed by an anhydrone trap.

Oxygen carries the formed $\mathrm{CO}_{2}$ to a molecular sieve trap at room temperature when it is collected. Follow- ing collection of the $\mathrm{CO}_{2}$, the trap is heated to approximately $600^{\circ} \mathrm{C}$ to release it. The second oxygen carrier system is directed through a system of solenoids at a flow rate of $500 \mathrm{cc} / \mathrm{min}$ to a measuring thermistor. The output of the thermal conductivity cell is integrated and read directly as \%C on an electronic digital voltmeter. With pure oxygen in the system the thermal conductivity cell is balanced to yield 0.000 output.

The temperatures of the thermistors are always higher than ambient oven temperatures in which the cell is located. This virtually eliminates normal room temperature variations.

The two thermistors of the conductivity cell are mounted in a metal block. When oxygen in one thermistor cavity is replaced by $\mathrm{CO}_{2}$, which has a lower thermal conductivity than oxygen, that thermistor becomes hotter and lower in electrical resistance. This electrical resistance change is calibrated to read percent $\mathrm{CO}_{2}$ in oxygen. For detailed electronics of model 761100 , see the LECO manual.

Refer to the Initial Reports of the Deep Sea Drilling Project, Volume 9 (Boyce and Bode, 1972) for further information regarding precision testing.

\section{REFERENCES}

Bader, R.G., Gerard, R.D., et al., 1970. Initial Reports of the Deep Sea Drilling Project, Volume 4: Washington (U.S. Government Printing Office).

Boyce, R.E. and Bode, G.W., 1972. Carbon and Carbonate Analysis, Leg 9, Deep Sea Drilling Project. In Hays, J.D., et al., Initial Reports of the Deep Sea Drilling Project, Volume 9: Washington (U.S. Government Printing Office), p. 797. 